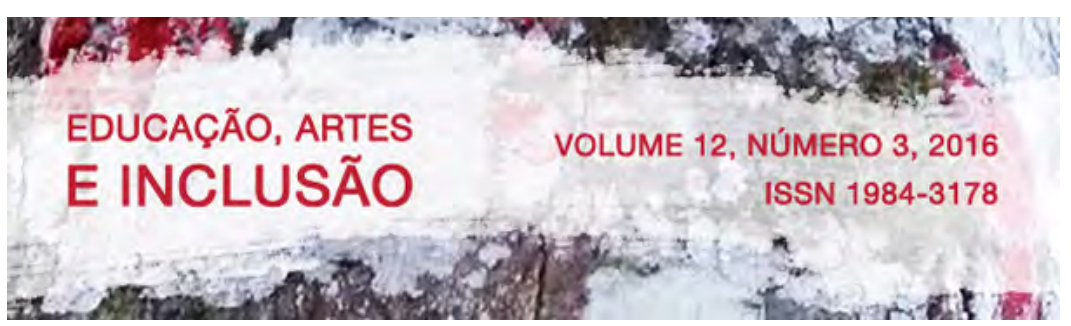

\title{
AÇÃO PEDAGÓGICA DE PREVENÇÃO ÀS PRÁTICAS RACISTAS NA ESCOLA: A PERCEPÇÃO SOBRE RACISMO ENTRE ESTUDANTES DO SEXTO ANO DO ENSINO FUNDAMENTAL
}

\section{ACTION TO PREVENT RACIST PRACTICES IN SCHOOL: THE PERCEPTION ON RACISM AMONG STUDENTS OF THE SIXTH DEGREE IN BRAZILIAN ELEMENTARY SCHOOL}

DOI: http://dx.doi.org/10.5965/1984317812032016086

Marcos Jorge - UNESP

\begin{abstract}
RESUMO
O artigo é resultado de um projeto desenvolvido na disciplina de História no sexto ano do Ensino Fundamental em uma escola da cidade de Bauru (SP) que discutiu o racismo e temas da cultura africana e afro-brasileira na esteira da Lei 10.639/03, que tornou obrigatório seu estudo no currículo da Educação Básica. Privilegiou-se a discussão da questão racial e, em particular, as manifestações do racismo escolar. A participação dos alunos foi ativa com muito entusiasmo, principalmente quando as atividades se estruturavam em torno de manifestações culturais, que são próximas da cultura infantojuvenil com músicas em ritmo de rap e funk, abordando, numa linguagem que lhes é habitual, o tema do racismo. Verificou-se que os estudantes possuem relativa consciência da diversidade étnica e cultural brasileira, não apenas em relação aos brancos e negros, mas também conseguem identificar as outras tantas etnias/culturas que formam o povo brasileiro - japoneses, indígenas, árabes, verificou-se que os alunos tendem a relacionar o racismo ao bulliyng, muitos demonstraram consciência do racismo como um crime e em várias oportunidades transcenderam as discussões para outras formas de preconceitos que vivenciam no dia a dia como: a obesidade, o modo de vestir/cortar cabelo e suas formas de comunicação.
\end{abstract}

PALAVRAS-CHAVES: Política educacional. Lei 10.639/03. Racismo.

\begin{abstract}
The article is the result of a project developed in the subject of history in sixth degree in brazilian elementary school in the city of Bauru (SP) which discussed racism and issues of African and AfricanBrazilian Culture, to accord the Law 10.639 / 03, has required its study on Basic Education curriculum. The discussion of the racial question is privileged and, in particular, the manifestations of racism in school daily. The participation of students was active, especially when the activities are structured around cultural events, which are common to the youth culture, with music and rhythm of rap and funk, a language which they usual. It was found that students have relative awareness of brazilian ethnic and cultural diversity, not only in relation to white and black, people but can also identify the many other ethnicities / cultures that form the brazilian heritage, peoples like a japanese, indians, arabs. The students usually to connect racism to bulliyng, many have shown awareness of racism as a crime and on several occasions transcended discussions to other forms of prejudice they experience on a daily life such as: obesity, forms of how to dress, models of haircuts and their forms of communication.
\end{abstract}

KEYWORDS: Educational policy. Brazilian educational law 10.639/03. Racism. 


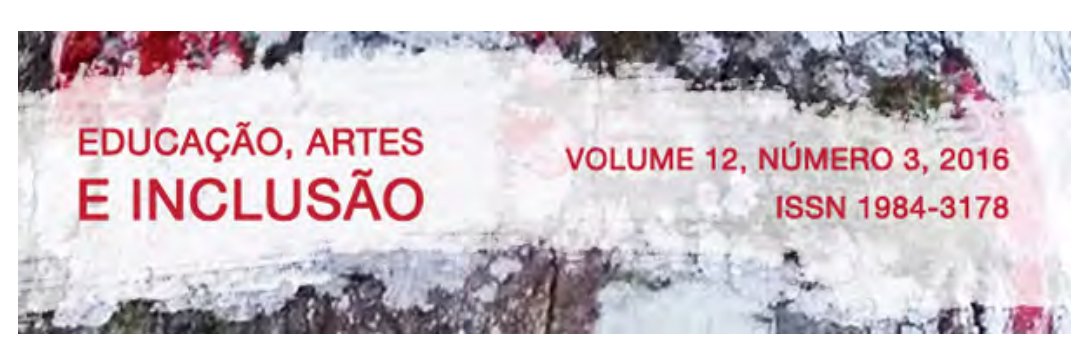

Assim, o sistema educacional brasileiro comporta uma grande contradição: enquanto ainda não universalizado com qualidade gera e reproduz desigualdades; no entanto é possível que esse mesmo sistema venha a contribuir para romper ciclos geracionais de marginalização da população mais pobre oferecendo uma educação de qualidade a todos que nele ingressem.

É nesse contexto de maior politização da questão racial e sua relação com a educação escolar que temas, como: a África, africanidade, negritude e ancestralidade, todos com a preocupação de valorização da identidade negra, africana e afro-brasileira foram incorporados às políticas públicas e o sistema educacional tornou-se uma arena de disputas para o resgate e a valorização da história e da cultura negra. (SALES JR., 2009).

Embora o racismo seja um traço cultural arraigado nas relações sociais brasileiras o recorte que discutiremos é o racismo na escola, que reflete em grande medida o fenômeno disseminado por toda a sociedade. Estudos como de Verçosa (2012) analisou o racismo escolar e afirma que o sistema educacional brasileiro é extremamente "discriminatório e pouco atraente para a população negra, trazendo consequências enormes em relação à escolarização levando muitos dos alunos a não concluírem as etapas de ensino ou a retardarem os anos de escolaridades”. (VERÇOSA, 2012, p.31)

A autora enfatiza que uma das consequências para a população negra é a impossibilidade de uma "mínima ascensão social" dos indivíduos, já que são tratados de forma discriminatória, concluindo que a escola deve participar ativamente para acabar com as desigualdades de oportunidades educacionais que estão vinculadas à questão racial.

Ainda nessa direção, Reis (2012) analisando o mesmo racismo escolar encontrou respostas contraditórias que mostram a complexidade do tema entre crianças e adolescentes em idade escolar. Destaca que uma maioria de respondentes do seu universo pesquisado (93\%) afirma não existir racismo na escola mas quando indagados sobre disseminar ou ouvir expressões pejorativas de cunho racial, outra grande maioria (95\%) respondeu afirmativamente; também foi verificada grande desinformação sobre conceitos de raça e etnia, diversidade, multiculturalismo e tolerância entre alunos e professores e equipes pedagógicas.

Este estudo chama a atenção para a urgência de "usar o espaço escolar para promover discussões sobre o racismo, a fim de promover uma reflexão e conscientização e para a desconstrução de estereótipos disseminados na cultura escolar” e expõe o caráter contraditório 


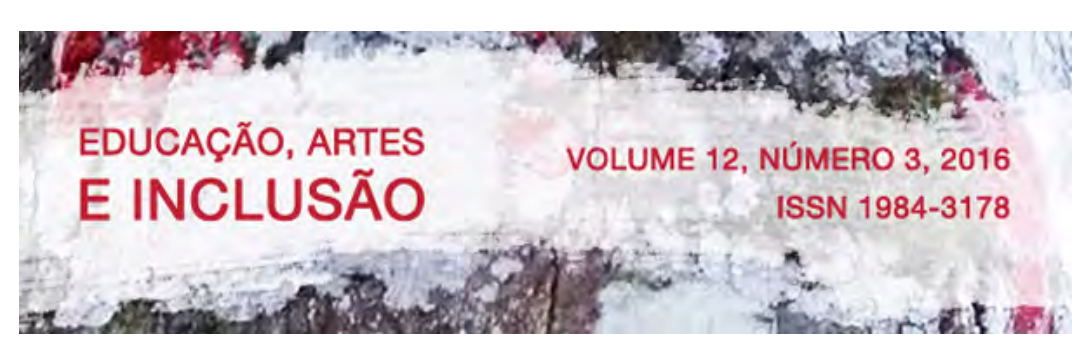

que a realidade escolar comporta em relação ao racismo negando-o e praticando-o simultaneamente. (REIS 2012, p.19).

Em vista do exposto, nosso estudo discute a importância da aplicação da Lei 10.639/03 que torna obrigatório o ensino da História e Cultura Afro-Brasileira e Africana no currículo dos ensinos fundamental e médio. A lei foi regulamentada pelo Conselho Nacional de Educação (CNE) através da Resolução $n^{0}$ 1/04 e do Parecer CNE/CP 3/04, esses documentos institucionalizaram as Diretrizes Curriculares Nacionais para a Educação das Relações Étnico-Raciais e para o Ensino de História e Cultura Afro-Brasileira e Africana nos sistemas de ensino.

Atualmente a dificuldade centra-se em fazer a lei ser aplicada nas escolas, nesse sentido Vergulino et. al. (2012) apontam alguns problemas entre eles o currículo dos cursos de formação de professores, enfatizando que:

infelizmente nos cursos de Pedagogia e Licenciatura, percebe-se muitas resistências sobre a inclusão da temática sobre a África e as questões da cultura afro-brasileira no ensino. Na maioria das grades curriculares dos cursos de graduação e pós graduação da área da educação, a África e as questões raciais brasileiras continuam invisíveis. (VERGULINO Et. Al., 2012, p. 8).

Por outro lado, Felipe e Teruya (2012) analisando representações de professores sobre o racismo encontraram a forte presença da crença na "democracia racial", na positividade da miscigenação e um discurso que relaciona o tema com a questão social. Tais posturas, segundo os autores obscurecem a compreensão dos professores para a necessidade da Lei $n^{\circ}$ 10.639/03, uma vez que se não há a percepção do racismo "também não há necessidade de uma lei para combater ou questioná-lo em sala de aula". (FELIPE e TERUYA, 2012, p. 211).

Os pontos aqui colocados mostram as dificuldades de trabalhar as Diretrizes Curriculares Nacionais para a Educação das Relações Étnico-Raciais e para o Ensino de História e Cultura Afro-Brasileira e Africana na escola, bem como outras variáveis que dificultam a sua aplicação de maneira mais imediata e sistemática como a resistência de segmentos escolares e mesmo pais de alunos. No entanto, diversas parcerias são possíveis no 


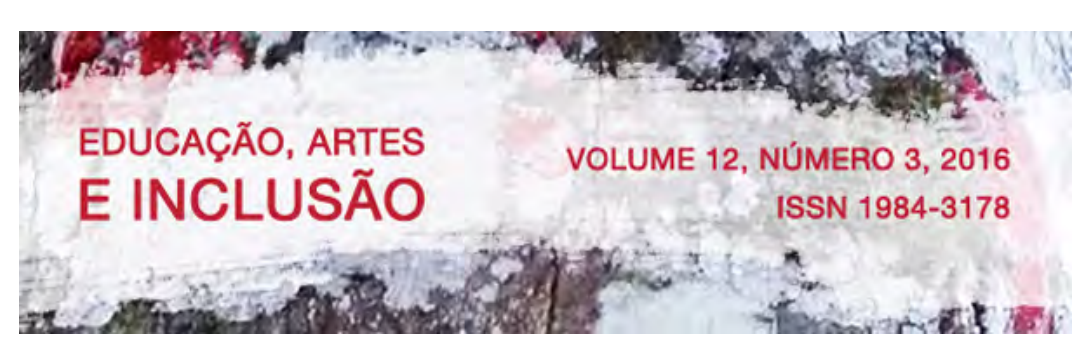

enfrentamento dessas resistências como as parcerias entre universidade e escola para realizar esse debate e um exemplo é o projeto: Ação pedagógica de prevenção às práticas racistas na escola que explicitaremos a seguir.

\section{AÇÃo PEDAGógiCA de PREVENÇÃo ÀS PRÁTICAS RACISTAS NA ESCOLA}

O projeto teve como objetivo disseminar conhecimentos e informações sobre a cultura africana e afro-brasileira e focou suas atenções na discussão do racismo promovendo debates e ações junto aos alunos dos anos finais do Ensino Fundamental ( $6^{\circ}$ ano) em uma escola pertencente a uma comunidade carente de um município do interior paulista. Foram utilizadas as aulas de história com o suporte de filmes documentários e ficção, leituras de diversos tipos de textos (revistas, músicas, historinhas em quadrinhos) e as ações envolveram discussões em rodas de conversas e atividades em que os alunos escreviam pequenas redações sobre o tema.

Inicialmente, discutiu-se a imagem da escola como microcosmo onde os jovens vivem parte de sua vida cotidiana, estabelecendo relações entre seus iguais e os segmentos que compõem a vida escolar e com a comunidade. Assim, a vivência nesse ambiente contraditório de regulação e espontaneidade, onde as relações alternam-se entre as mais livres e descontraídas até aquelas marcadas pelo formalismo exigido pela instituição escolar foi bastante explorada nas rodas de conversas.

Nesse universo marcado por ambivalências os conflitos ocorrem diariamente e as manifestações explícitas ou implícitas de racismo estão presentes nesta "cultura escolar" brasileira. Desta forma, problematizou-se a rotina da escola, seus segmentos e o entorno local, entendendo-os como elementos importantes na prevenção do racismo; mais importante foi o estabelecimento de uma metodologia amparada nos códigos e registros culturais que fazem parte da cultura juvenil como a música, o cinema, os jogos, as brincadeiras e as artes.

Um dos objetivos foi despertar uma consciência do chamado "racismo cordial" utilizando o aporte de letras e músicas, principalmente aqueles ritmos mais apreciados e consumidos pelas crianças atendidas pela escola, como o rap, o funk e o hip hop, mas também porque expressam temas que lhe são muito próximos, como a violência urbana, as subculturas juvenis, a marginalidade social e o racismo. 


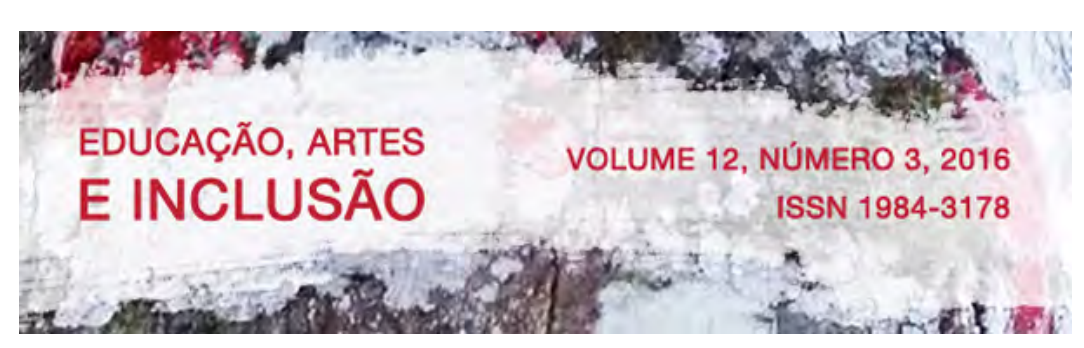

Foram feitas atividades lúdicas de expressão artística com desenhos, pinturas, recortes, colagens e composições acompanhadas de discussão dos elementos que apareciam nos registros escritos produzidos pelas crianças. Ao final, grandes resultados foram alcançados como uma maior relação de confiança entre os bolsistas e os alunos e observou-se uma maior participação dos mesmos com um comportamento de respeito, cordialidade e seriedade.

Finalmente, os estagiários estimularam a discussão com a proposição de trabalhos livres e dentro de uma atmosfera de descontração na sala de aula e em outros espaços da escola, com o andamento do projeto as crianças sentiram-se mais livres e foi possível buscar outras formas de expressão como pequenas encenações teatrais, filmagens com celulares e filmadoras e pesquisa de vídeos/videoclipes na Internet que eram exibidos na sala e serviam para ampliar as discussões.

\section{ESTRATÉGIAS E DINÂMICAS DE CONSCIENTIZAÇÃO}

Para a interpretação dos registros escritos foi utilizada a metodologia da análise de conteúdo (AC) que consiste em uma técnica de análise de dados qualitativa, usada com muita frequência nas ciências sociais, que procura examinar as dimensões subjetivas individuais ou de grupo na percepção de um fenômeno social e sua relação com a realidade.

A AC segundo Bardin (2009) possui dois objetivos: o primeiro é a "superação da incerteza", a que está sujeita toda pesquisa qualitativa e o segundo é o "enriquecimento da leitura" dos dados disponíveis, a técnica pode ainda ser aplicada para as pesquisas com dados quantitativos ou para aquela delineada partir somente de dados qualitativos; a mudança está no tratamento dos dados:

$\mathrm{Na}$ análise quantitativa, o que serve de informação é a frequência com que surgem certas características do conteúdo. Na análise qualitativa é a presença ou ausência de uma dada característica de conteúdo ou de um conjunto de características num determinado fragmento de mensagem que é tomada em consideração. (Bardin, 2009, p.22) 


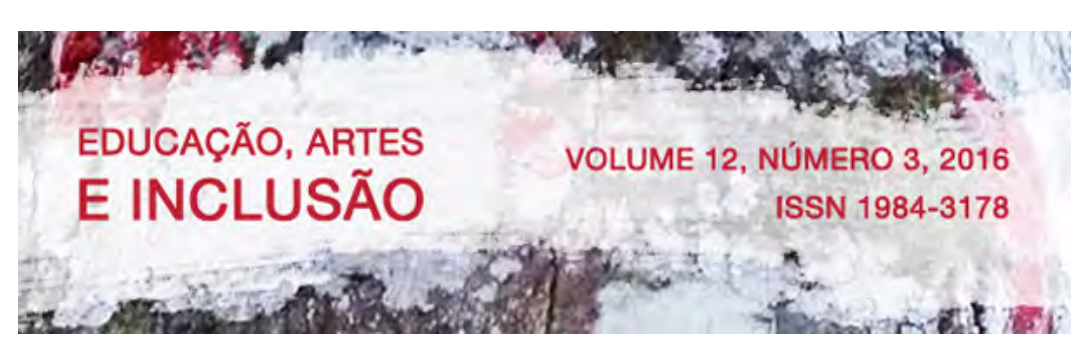

Câmara (2013) fornece outras pistas metodológicas para a realização da AC, afirma que é preciso seguir determinados passos que se constituem como regras do método enfatizando que é necessário ao pesquisador:

\begin{abstract}
obedecer às regras de exaustividade (deve-se esgotar a totalidade da comunicação, não omitir nada); representatividade (a amostra deve representar o universo); homogeneidade (os dados devem referir-se ao mesmo tema, serem obtidos por técnicas iguais e colhidos por indivíduos semelhantes); pertinência (os documentos precisam adaptar-se ao conteúdo e objetivo da pesquisa) e exclusividade (um elemento não deve ser classificado em mais de uma categoria). (CÂMARA, 2013, p. 181).
\end{abstract}

Outra referência importante na discussão da AC é Silva e Fossá (2013) que estabelecem três momentos no uso da metodologia, o primeiro seria a "sistematização das ideias do quadro teórico e o estabelecimento de indicadores para a interpretação das informações", o segundo trata da "construção das operações de codificação, considerando-se os recortes dos textos em unidades de registros, a definição de regras de contagem e a classificação e agregação das informações em categorias simbólicas" e o último seria o momento de "captar os conteúdos manifestos e latentes ressaltando os aspectos considerados semelhantes e os que foram concebidos como diferentes". (SILVA e FOSSÁ, 2013, p. 3-4),

Os registros escritos dos alunos contemplaram aquelas regras e representaram a totalidade de uma mesma turma do sexto ano do ensino fundamental, mantiveram a homogeneidade e atenderam à regra da exclusividade. A partir desses elementos, selecionouse o material escrito pelas crianças para uma análise a partir de categorias que foram definidas por Silva e Fossá (2013) como sendo aquelas "estruturas simbólicas ou temáticas que auxiliam na compreensão do que está por trás dos discursos", as categorias que utilizamos foram: consciência do racismo, a cor da pele, igualdade e diferença. (SILVA e FOSSÁ, 2013, p.2).

\title{
4 ENQUANTO A COR DA PELE É MAIS IMPORTANTE QUE O BRILHO DOS OLHOS, SEMPRE VAI EXISTIR GUERRA
}

\subsection{Consciência do racismo}




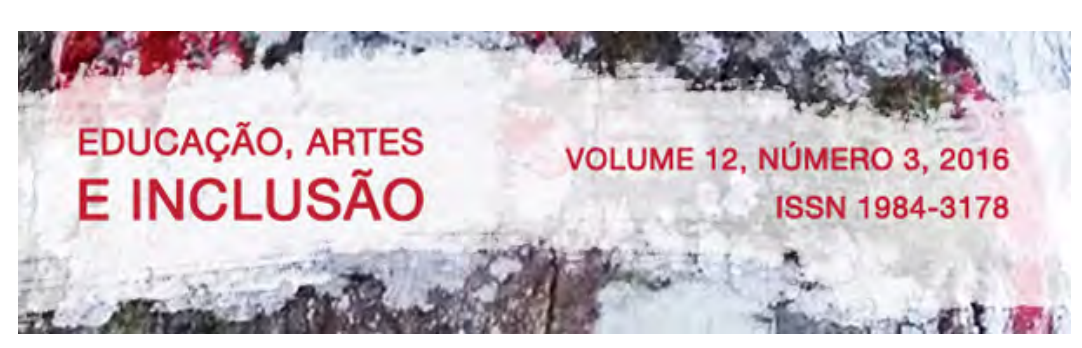

Na escola, o racismo está ausente das discussões em sala de aula; geralmente o que é "falado/contado na escola, sobre os povos que compõem a sociedade brasileira, é incompleto, lacunar, estereotipado, quando não omitido/negado". Por outro lado, ao não problematizar a questão racial a escola contribui para manter a percepção errônea da sociedade brasileira, difundindo a imagem da "democracia racial", ou propiciando condições para que a cultura escolar reproduza, por meio do racismo institucionalizado, os preconceitos raciais. (TRINDADE, 2002, p.34).

Assim, os registros mostram que os alunos têm uma consciência do racismo de maneira abrangente e alguns trazem uma percepção mais contundente da sua existência no locus escolar como fenômeno que transcende a pessoa negra e que atinge outros grupos da população, os alunos entendem também que o racismo é uma forma de violência:

Nessa escola existe muito preconceito e racismo e eu acho que tinha que parar com essas coisas por que somos todos iguais. (aluno 5).

Preconceito não se deve fazer, racismo também não. (aluna 14).

$O$ racismo parte da premissa de que alguém é superior. O negro é sempre inferior. E dessa pessoa não se admite sequer que ela abra a boca... (aluno 7).

Somos todos iguais branco, negro, gordo e magro. (aluno 10).

O que o branco tem, que o preto não tem? O que é melhor no magro, que o gordinho não pode ter? Enquanto a cor da pele for mais importante que o caráter haverá guerra no mundo. (aluna 7).

Eu não gosto do bullyng, do racismo e do preconceito, porque machuca as pessoas. (aluna 12)

O racismo não é legal porque machuca o sentimento das pessoas. (aluno $1)$.

\subsection{A cor da pele}

Segundo Silva (2012) no Brasil:

as expressões relacionadas à cor e à raça são construídas de modo relacional e situacional. A utilização de termos como negro, mais escuro, preto, mulato ou termos vexatórios, como crioulo, é relacionado a afinidades existentes entre os interlocutores, à posição social, à faixa etária e a valores, como respeito e autoridade (SILVA, 2012, p.9). 


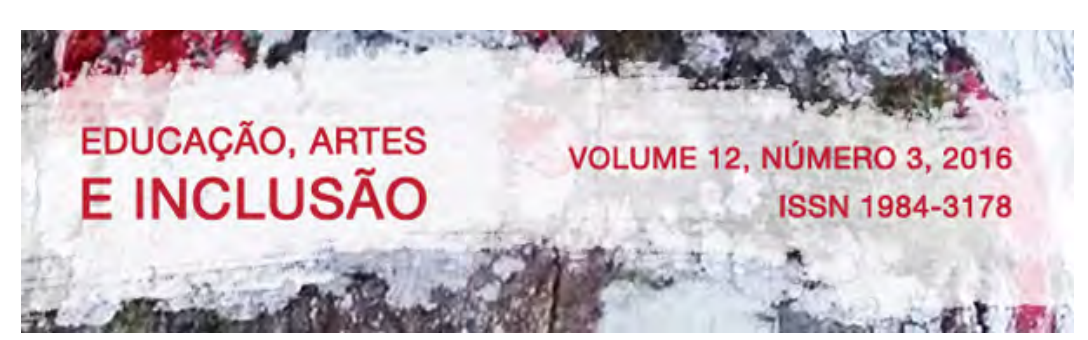

Essa autora aponta para uma situação tipicamente brasileira, em que "temos um racismo em gradação que atinge em maior escala aqueles que mais se aproximam do fenótipo negro, esse sistema é motivado por fatores históricos, sociais e políticos" e assim constrói-se um racismo muito peculiar já que não se apoia apenas no fenótipo agregando também o preconceito de classe por isso ela afirma que o racismo brasileiro não atinge, da mesma forma, outras etnias como árabes e asiáticos. Finalmente, a origem social pobre dos indivíduos associada ao fenótipo negro, mulato, moreno ou qualquer outra denominação associada à cor da pele não branca torna-se um dos fatores mais fortes à manutenção desse racismo brasileiro. (SILVA, 2012, p.10).

Nesse sentido, percebemos que a cor da pele destaca-se como um viés recorrente na percepção do racismo pois sugere haver uma consciência muito forte de que a questão racial está vinculada ao grupo populacional negro. As referências à cor aparecem associadas à ideia de disseminação do racismo, de que este constitui bullyng e é um crime, seguidas de argumentos a favor da cor da pele como algo secundário para a qualidade das relações humanas.

Somos todos iguais, mesmo tendo diferenças de cor e racismo é bully (sic) e racismo é crime. (aluna 8 ).

Não julgue as pessoas pela capa (sic) ou pela cor, mas sim pelo caráter. Diga não ao racismo. (aluno 6).

Ninguém é diferente de ninguém somos todos iguais, quando o caráter for mais importante que a cor da pele, o mundo irá evoluir. (aluna 4). Não importa a cor, o importante é amar (aluno 13).

Não julgue pela cor, não julgue pela aparência, todos são iguais. (aluno 12).

Ignorar o que falam e não dar atenção para o que os outros falam da sua cor. (aluno 11).

Feliz será o dia em que todos os homens aceitarem o que não importa a cor da pele e sim a humildade. (aluna 1).

Enquanto a cor da pele é mais importante que o brilho dos olhos, sempre vai existir guerra. (aluna 5).

Não existem raças, existem pessoas. (aluno 9).

Racismo é crime mas tem que ter consciência, por favor, sem ofensa. (aluna 6). 


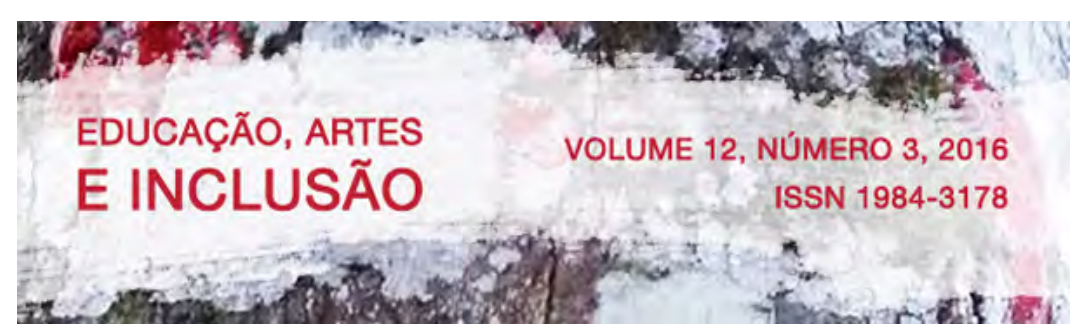

\subsection{Igualdade e diferença}

Não discutiremos a questão dos princípios políticos iluministas e das tradições políticas posteriores sobre a igualdade de direitos, ainda que formais, e que formam a base da vida moderna amparada por direitos. No que concerne a este trabalho, essas questões aparecem nos relatos numa relação de reciprocidade; percebe-se uma chamada à consciência de que a humanidade é uma entidade única e que o racismo é uma nódoa neste "estado de coisas".

As diferenças ou os "defeitos" que todos portam aparecem como parte das pessoas e isto é sentido como algo que acomete a todos sem distinção e essas diferenças são constituintes dos seres humanos e devem ser entendidas como características únicas que distinguem cada pessoa. Trabalhando essa relação, Candau (2008, p. 46) afirma que em relação à igualdade, atualmente há um "forte deslocamento, não se nega a igualdade, mas se coloca muito mais em evidência o tema da diferença" e essa tendência apareceu nas discussões durante o desenvolvimento do projeto.

Os registros revelam que há uma percepção de que existe uma igualdade entre todos (na condição de seres humanos) mas também que há diferenças e estas não podem ser condição de desqualificação das pessoas.

As referências ao estilo do cabelo, aos "defeitos" de cada um e um "fim" que nos aguarda a todos indistintamente aparecem como referenciais das crianças para repudiar o racismo e expressar um desejo de igualdade real entre as pessoas e toda humanidade.

\footnotetext{
Meu cabelo não é ruim, ruim é o seu racismo. (aluna 2).

Olhe os seus defeitos pra depois falar das pessoas! Diga não ao preconceito. (aluna 13).

Ninguém é diferente de ninguém

Somos todos iguais por isso

Não devemos maltratar e nem julgar. (aluna 3).

Todos somos iguais, ninguém é melhor que ninguém. (aluna 9).

Para que racismo? No final seremos a mesma coisa! (aluna 10).

Somos todos iguais, sem racismo. (aluna 11).

Chega de preconceito e racismo, somos humanos. (aluno 4).
}

A partir desses elementos cotejamos os dados com alguns autores que trabalharam no interior da escola em projetos que buscavam discutir o racismo no cotidiano escolar, a 


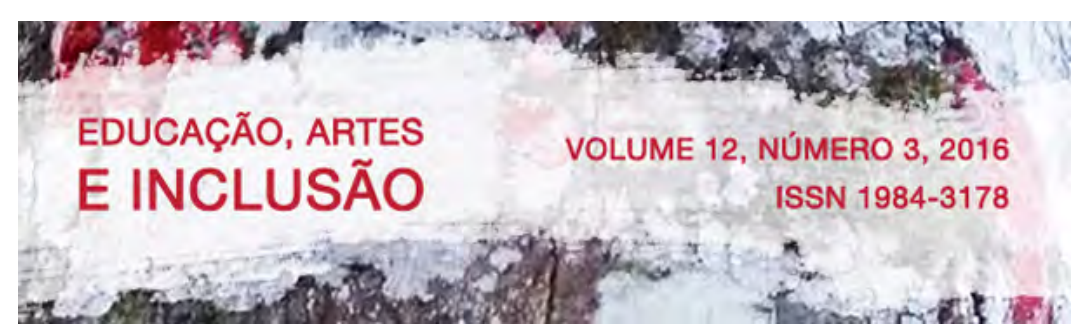

\section{CONSIDERAÇÕES FINAIS}

O projeto Ação Pedagógica de Prevenção às Práticas Racistas na Escola teve como objetivo difundir ações, ideias e valores que discutissem a questão racial brasileira nas aulas de História numa escola no interior de São Paulo. Para tanto, apoiou-se na Lei 10.639/03, que torna obrigatório o ensino da História e Cultura Afro-brasileira e Africana no currículo da escola básica e em outros documentos oficiais que institucionalizaram as Diretrizes Curriculares Nacionais para a Educação das Relações Étnico-Raciais e para o Ensino de História e Cultura Afro-Brasileira e Africana nos sistemas de ensino.

Neste sentido, o projeto buscou parcerias entre a escola, a instituição universitária e o seu departamento de educação e contou com a participação de dois bolsistas, que por serem universitários, jovens e não fazerem parte do quadro de pessoal da escola facilitou muito a relação de confiança e segurança com as crianças.

Pudemos verificar que as crianças têm relativa consciência da diversidade étnica brasileira, não apenas em relação aos brancos e negros mas também conseguem identificar as outras tantas etnias que formam o povo brasileiro como japoneses, indígenas e árabes. Em decorrência de outros projetos desenvolvidos na escola as crianças associaram o racismo ao bulliyng e demonstraram ter conhecimento de se tratar de um crime e que deve ser denunciado. Um ponto importante foi constatar que ao romper com o clima de formalismo da escola as crianças se expressaram com muita espontaneidade e relataram sem timidez acontecimentos do seu cotidiano reforçando a confiança mútua e o respeito entre os colegas.

Finalmente, entendemos que o projeto atingiu seus objetivos ao levar para a escola a discussão sobre o racismo e a diversidade étnica brasileira contribuindo para adentrar a escola com temáticas que são urgentes no campo da educação básica. 


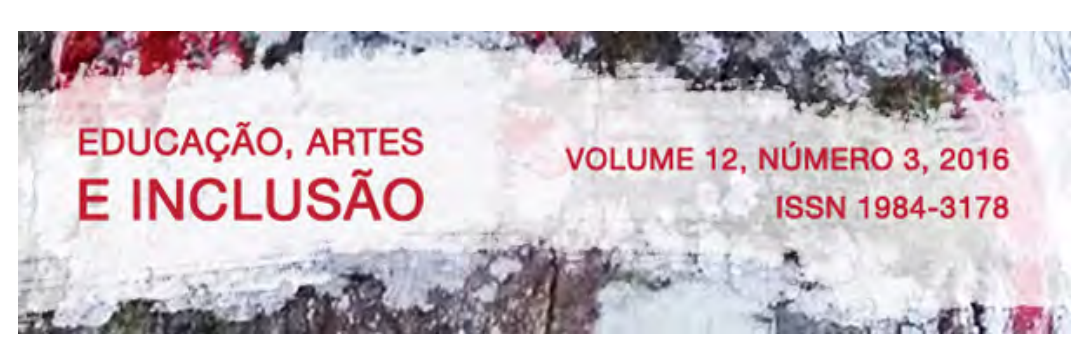

ORTIZ, Cisele. Só não enxerga quem não quer: Racismo e preconceito na Educação Infantil. Revista Avisalá, no 23, nov. 2005.

REIS, Deyse Almeida. Uma reflexão sobre o racismo no âmbito escolar. Monografia (Especialização). Programa de Pós-Graduação em Educação para a Diversidade da Universidade Federal de Ouro Preto. Instituto de Ciências Humanas e Sociais - UFOP, 2012.

RIBEIRO, Carlos Antonio Costa. Desigualdade de Oportunidades e Resultados Educacionais no Brasil. DADOS - Revista de Ciências Sociais, Rio de Janeiro, vol. 54, no 1, 2011, pp. 41 a 87.

SALES JR., Ronaldo L. Políticas de Ancestralidade: negritude e africanidade na esfera pública. CAOS - Revista Eletrônica de Ciências Sociais, n. 14, Setembro/2009. Disponível em: < www.cchla.ufpb.br/caos>. Acesso em 12-abr-2015.

SILVA, Francisca Cordelia Oliveira. Etnia, cor e raça: aspectos discursivos do uso institucional. 2012. Disponível em: $<$

http://www.fflch.usp.br/dlcv/enil/pdf/64_Francisca_Cordelia_OS.pdf $>$ Acesso em: 10-mar2014.

Andressa Hennig. FOSSÁ, Maria Ivete Trevisan. Análise de Conteúdo: Exemplo de Aplicação da Técnica para Análise de Dados. IV Encontro de Ensino e Pesquisa em Administração e Contabilidade - ENEPQ. Brasília - DF - 3 a 5 novembro de 2013. Anais.

TRINDADE, Azoilda Loretto. O racismo no cotidiano escolar. Dissertação (Mestrado). Fundação Getúlio Vargas Instituto de Estudos Avançados em Educação Departamento de Psicologia da Educação, 2002.

UNICEF. Fundo das Nações Unidas para a Infância. Acesso, permanência, aprendizagem e conclusão da educação básica na idade certa - Direito de todas e de cada uma das crianças e dos adolescentes. - Brasília: UNICEF, 2012. "Iniciativa Global Pelas Crianças Fora da Escola - Brasil".

VERGULINO, Ana Rosa et.al. Relações étnico-raciais no espaço escolar. Revista Interação v.1, n. 2, 2012. Disponível em:<

http://www.portalamericas.edu.br/revista/pdf/ed12/artigo1.pdf $>$. Acesso em 02-set-2014.

VERÇOSA, Alzenite de Araújo. Racismo na escola: o silêncio fala mais alto. Monografia (Especialização). Programa de Pós-Graduação em Educação para a Diversidade da Universidade Federal de Ouro Preto. Instituto de Ciências Humanas e Sociais - UFOP, 2012. 